\title{
miR-129-5p ameliorates ischemia-reperfusion injury by targeting HMGB1 in myocardium
}

\author{
Jingxian Xing, Junyan Liu, Juan Liu and Zesheng Xu \\ Second Department of Cardiovascular Medicine, Cangzhou Central Hospital, Cangzhou, Hebei, People's Republic of China
}

\begin{abstract}
Globally, acute myocardial infarction (AMI) is a serious condition affecting millions of individuals. While AMI therapy improves blood flow during surgery, reperfusion-induced injury may also occur, leading to secondary cardiac damage or even death. Here, we investigated miR$129-5 \mathrm{p}$ in myocardial ischemia-reperfusion (I/R) injury in rats, to explore reperfusion-related molecular mechanisms in myocardium. We used Sprague Dawley rats to establish a myocardial I/R model, with agomiR-129-5p injection, and used rat cardiomyocytes (H9c2) treated with anoxia-reoxygenation $(\mathrm{A} / \mathrm{R})$ to mimic myocardial $\mathrm{I} / \mathrm{R}$ injury in vitro. A dual-luciferase reporter assay determined miR-129-5p binding to high mobility group box-1 (HMGB1) in H9c2 cells. We showed that exogenous miR-129-5p restored cardiac function indices, alleviated cardiac injury, relieved inflammatory effects and reduced infarct size and cell apoptosis in rat myocardium after I/R treatment. Elevated miR-129-5p induced a reduction in HMGB1 expression in rat I/R myocardium. miR-129-5p also targeted HMGB1, and negatively regulated its expression in H9c2 cells. Moreover, miR-129-5p overexpression in the cardiomyocytes reduced cell apoptosis and recovered cell viability after $A / R$ injury, which was reversed by subsequent HMGB1 overexpression. These findings suggest miR-129-5p plays a cardioprotective role in ameliorating myocardial I/R injury in rats, by negatively targeting HMGB1. This mechanism provides new insights into the treatment of myocardium reperfusion-related damage.
\end{abstract}

Key words: Myocardial reperfusion - I/R injury - miR-129-5p - HMGB1 - Myocardial infarction

\section{Introduction}

Acute myocardial infarction (AMI) manifests as myocardial necrosis, due to acute myocardial ischemia accompanied by acute myocardial injury. The condition is characterized by dysfunctional cardiac biomarkers, which contribute to significant morbidity and mortality (Thygesen et al. 2018). Annually, AMI affects the health and life quality of more than seven million individuals, on a global level (Reed et al. 2017). Blood flow improvement or myocardial reperfusion via antithrombotic therapies or primary percutaneous coronary intervention (PCI), or combinations thereof, are effective strategies in limiting myocardial infarct size and improving clinical outcomes for AMI patients (Yellon and Hausenloy

Correspondence to: Zesheng Xu, Second Department of Cardiovascular Medicine, Cangzhou Central Hospital, 16 West Xinhua Road, Cangzhou, Hebei 061001, People’s Republic of China E-mail: xzsdoctor@126.com
2007; Reed et al. 2017). However, myocardial reperfusion injury often occurs, causing extra myocardium damage or even death (Yellon and Hausenloy 2007). Although ischemic conditioning during myocardial reperfusion is a useful cardioprotective strategy in recent decades, the numbers of subsequent heart failure episodes and other comorbidities have not declined (Hausenloy and Yellon 2016). Therefore, the amelioration of myocardial ischemia-reperfusion (I/R) injury is of particular clinical interest.

Endogenous microRNAs (miRNAs, 22 nucleotides) have been studied extensively due to their powerful biological regulation of target genes at transcriptional and post-transcriptional levels, and are associated with various disease processes (Ha and Kim 2014; Pu et al. 2019). In heart physiology, multiple miRNAs are aberrantly expressed during myocardial infarction (MI) and MI-related protective mechanisms, and are regarded as potential biomarkers or therapeutic targets for cardiac diseases (Colpaert and Calore 2019; Kura et al. 2020). The identification and charac- 
terization of novel miRNAs implicated in cardiac regulation could uncover underlying molecular mechanisms of MI and myocardial reperfusion injury (Kura et al. 2020). Recently, in myocardial cells, miR-129-5p was shown to inhibit hydrogen peroxide $\left(\mathrm{H}_{2} \mathrm{O}_{2}\right)$-induced cell autophagy and apoptosis, via a PI3K/Akt/mTOR signaling circuit, by targeting ATG14 (Zhang et al. 2018). Moreover, Li et al. (2017) demonstrated that miR-129-5p was downregulated after spinal cord I/R injury in mice, whereas miR-129-5p overexpression ameliorated I/R-induced neuro-inflammation and blood-spinal cord barrier damage by directly targeting the cytokine, high mobility group box-1 (HMGB1). Equally, miR-129-5p was also shown to protect against hypoxia-ischemia brain injury, through distinct targets in neonatal mice and rats (Zhou et al. 2018a, 2018b). However, the effects of miR-129-5p on myocardial I/R injury have yet to be reported.

HMGB1 is a binding target of miR-129-5p (Li et al. 2017; Liu et al. 2017a), with two main functions; the specific structure of HMGB1 confers its function as a transcription factor to bind to and bend DNA to assemble nucleoprotein complexes, and HMGB1 also serves as an extracellular growth mediator with crucial roles in inflammation and wound repair (Ranzato et al. 2012; Lee et al. 2015). Previously, the expression of HMGB1 was shown to be upregulated during I/R injury, either at the heart or liver, whereas HMGB1 downregulation alleviated I/R injury and also its following inflammatory response (Andrassy et al. 2008; Dong et al. 2018; Xie et al. 2018). This evidence suggests that miR-129-5p may also ameliorate myocardial I/R injury by targeting HMGB1, therefore we tested this hypothesis in this study.

\section{Materials and Methods}

\section{Animals}

Eight-week-old male Sprague Dawley (SD) rats (Liaoning Changsheng Biotechnology Co., Ltd., China) were used for in vivo work. All animals were kept in a $12 \mathrm{~h}$ light/dark cycle at a temperature of $22 \pm 1^{\circ} \mathrm{C}, 45-55 \%$ humidity with free access to food and water for one week of adaptive feeding. All animal experiments were approved by the Ethics Committee of Cangzhou Central Hospital and all experimental protocols were performed following the Guide for the Care and Use of Laboratory Animals (National Research Council Committee for the Update of the Guide for the and Use of Laboratory 2011).

\section{Animal modeling and myocardial I/R injury}

Animals were randomly divided into four groups: Sham, I/R, I/R+agomiR-NC (negative control), and I/R+agomiR-129-5p
(GenePharma, Shanghai, China). Each group contained six animals. For in vivo transfection of miR-129-5p reagents, a left thoracotomy was performed on rats and agomiR-NC or agomiR-129-5p (0.5 nmol per animal) was mixed with Entranster ${ }^{\mathrm{TM}}$-in vivo $(8 \mu \mathrm{l})$ (Engreen Biosystem Co., Ltd., Beijing, China), and injected into the anterior wall of the left ventricle of rat myocardium, at six random points (Fu et al. 2017). Rats in the Sham and I/R groups were injected with an equal amount of the transfection reagent. After suturing the wound and a four day recovery, rats were killed and myocardium was collected to examine miR-129-5p expression levels by real-time PCR.

Once miR-129-5p was confirmed as upregulated in relevant groups, the I/R injury model was instigated through left anterior descending (LAD) ligation surgery, after agomiR-129-5p (or agomiR-NC) re-injection into intact animals for four days. Briefly, rats were anesthetized by pentobarbital sodium using intraperitoneal injection (i.p.). After fixation, the neck trachea was incised and exposed to perform endotracheal intubation and ventilation. A left thoracotomy was carried out between the third and fourth intercostal space, and an MI model was formed by blocking blood flow in the anterior descending branch using a sterile 5-0 suture (Shanghai Yuanhong Medical Appliance Co., Ltd., China) ligation. Animals in the Sham group had similar procedures without tying the suture. After ligation for $45 \mathrm{~min}$, coronary artery blood flow was restored and reperfused for $3 \mathrm{~h}$. Cardiac function was then examined, and myocardium tissue and serum from different groups collected (Fig. 1). Some myocardium tissue was cryopreserved in liquid nitrogen and stored at $-70^{\circ} \mathrm{C}$, while other tissue was fixed in $4 \%$ paraformaldehyde for further experiments and 2, 3, 5-triphenyltetrazolium chloride (TTC, Solarbio, Beijing, China) staining, according to manufacturer instructions. The MI size in each sample after TTC staining was quantified using Image Pro-Plus (IPP) 6.

\section{Cardiac function measurement}

Rats were anesthetized by pentobarbital sodium $(50 \mathrm{mg} / \mathrm{kg}$ ) by i.p. Ultrasound of the left ventricle (LV) was performed using a GE Vivid 7 Dimension ultrasound apparatus (probe $\mathrm{M} 12 \mathrm{~S}$, frequency range 4-12 MHz). The ejection fraction (EF) and fractional shortening (FS) in each group were calculated. Then, rat neck was incised and the right carotid artery separated. After ligating the distal end, and clamping the proximal end, a polyethylene catheter (inner diameter: $0.5 \mathrm{~mm}$, outer diameter: $1 \mathrm{~mm}$ ), filled with $500 \mathrm{U} / 1$ heparin sodium and saline solution, was slowly inserted into the LV. Meanwhile, a pressure sensor was connected to the other end of the catheter to collect the data of left ventricular systolic pressure (LVSP) and left ventricular end-diastolic pressure (LVEDP). 


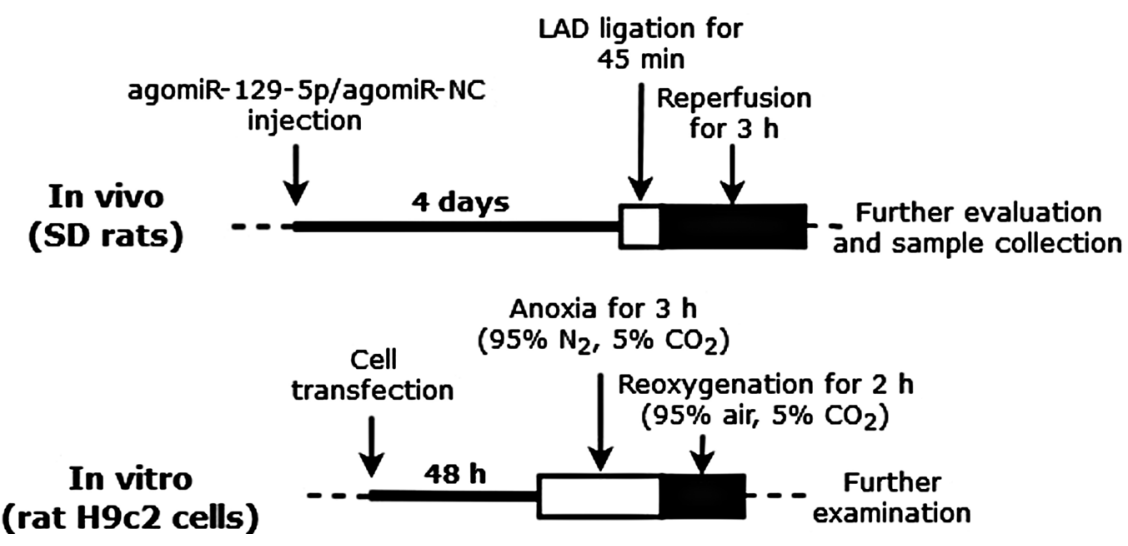

Figure 1. Experimental scheme outlining modeling procedures and timelines for in vivo and in vitro studies.

\section{Cell culture and cell transfection}

Rat myocardial H9c2 cells were purchased from Shanghai Zhongqiaoxinzhou Biotech (China), and cultured in Dulbecco's Modified Eagle Medium (DMEM, HyClone, Logan, Utah, USA) with $10 \%$ fetal bovine serum (FBS, Biological Industries, Beit-Haemek, Israel) at $37^{\circ} \mathrm{C}$ in $5 \% \mathrm{CO}_{2}$. One day before transfection, cells ( $\sim 90 \%$ confluency) were seeded in 6-well-plates, and cultured in serum-free medium for $1 \mathrm{~h}$, followed by Lipofectamine ${ }^{\mathrm{TM}} 2000$ transfection reagent (Invitrogen, Thermo Fisher Scientific, Carlsbad, CA, USA)mediated transfection based on manufacturer instructions. Briefly, H9c2 cells were transfected with 100 pmol agomiR129-5p/antagomiR-129-5p/NC or 50 pmol agomiR-129-5p + $1 \mu \mathrm{g}$ pcDNA3.1+ (Clontech, CA, USA) containing HMGB1 (or empty) in each well, with addition of $8 \mu$ l of Lipofectami$n \mathrm{e}^{\mathrm{TM}} 2000$. After transfection, cells were cultured at $37^{\circ} \mathrm{C}$ in $5 \% \mathrm{CO}_{2}$.

\section{Anoxia-reoxygenation $(A / R)$ injury in vitro}

$\mathrm{A} / \mathrm{R}$ treatment was conducted $48 \mathrm{~h}$ after transfection. Cells cultured in serum-free and glucose-free DMEM were incubated in $95 \% \mathrm{~N}_{2}, 5 \% \mathrm{CO}_{2}$ for $3 \mathrm{~h}$, after which media was replaced with complete DMEM containing 10\% FBS, and cells incubated in $95 \%$ air, $5 \% \mathrm{CO}_{2}$ for $2 \mathrm{~h}$. Afterward, cells were processed (Fig. 1).

\section{Enzyme activity and inflammatory marker analysis}

Serum creatinine kinase $(\mathrm{CK})$ and lactate dehydrogenase (LDH) activities were measured using $\mathrm{CK}$ and $\mathrm{LDH}$ assay kit, respectively (Nanjing Jiancheng Bioengineering Institute, China) according to manufacturer protocols. ELISA kits for serum interleukin-1 $\beta$ (IL-1 $\beta$ ) and tumor necrosis factor- $\alpha$ (TNF- $\alpha$ ) (Cloud-Clone Corp., Wuhan, China) determination were used in accordance with manufacturer instructions.
Immunofluorescence-TUNEL (IF-TUNEL) assay

Paraffin tissue sections were de-waxed and rehydrated by xylene and ethanol, respectively. Permeabilization was performed using $50 \mu \mathrm{l} 0.1 \%$ TritonX-100 (Beyotime, Shanghai, China), followed by $3 \% \mathrm{H}_{2} \mathrm{O}_{2}(50 \mu \mathrm{l})$ blocking treatment. A terminal deoxynucleotidyl transferase dUTP nick end labeling (TUNEL) reaction solution $(50 \mu \mathrm{l})$ was prepared according to the One Step TUNEL Apoptosis Assay Kit (Beyotime), to label samples with green fluorescence (Fluorescein isothiocyanate, FITC). DAPI (Biosharp, Hefei, China) was used for nuclear counter-staining. After mounting with antifade medium (Solarbio), sample images were observed and captured using a confocal microscope (Olympus, Tokyo, Japan) at $400 \times$ magnification.

\section{CCK-8 assay}

Cells were seeded $\left(3 \times 10^{3}\right)$ in 96 -well-plates, with five replicates for each group. After cell transfection and A/R treatment, the medium in each well was replaced with $100 \mu \mathrm{l}$ complete DMEM plus $10 \mu \mathrm{l}$ CCK- 8 (Sigma-Aldrich, Darmstadt, Germany). Cells were incubated at $37^{\circ} \mathrm{C}$ in $5 \%$ $\mathrm{CO}_{2}$ for $1 \mathrm{~h}$, after which optical density (OD) at $450 \mathrm{~nm}$ was measured on an ELX-800 absorbance reader (BioTek, Winooski, VT, USA).

\section{Cell apoptosis determination using flow cytometry}

The Annexin V-FITC apoptosis detection kit (Beyotime) was used to detect cell apoptosis in H9c2 cells. Briefly, cells in 6-well-pates from each group were centrifuged, collected, and incubated with $200 \mu \mathrm{l}$ Annexin V-FITC and $10 \mu \mathrm{l}$ propidium iodide (PI) for $15 \mathrm{~min}$ at room temperature in the dark, followed by incubation on ice. Cells were gently resuspended 2-3 times to improve staining, and were processed by flow cytometry (NovoCyte Flow Cytometers, ACEA Biosciences, San Diego, CA, USA). 


\section{Real-time PCR}

Total RNA was extracted using the high pure total RNA rapid extraction kit (BioTeke, Beijing, China), and reverse transcribed into cDNAs using M-MLV reverse transcriptase (Takara, Beijing, China) and RNase inhibitors (Takara). Oligo $(\mathrm{dT})^{15}$ and RT primer were used for HMGB1 and miR-129-5p amplification during reverse transcription reactions. The RT primer was: 5'-GTTGGCTCTGGTGCAGGGTCCGAGGTATTCGCACCAGAGCCAACGCAAGC-3'. Real-time PCR was performed with cDNA templates, primers (Genscript, Nanjing, China), SYBR GREEN (BioTeke), and Taq ${ }^{\text {TM }}$ HS Perfect Mix (Takara), using the Exicycler ${ }^{\text {TM }} 96$ Real-time Quantitative PCR system (Bioneer, Daejeon, Republic of Korea). Relative expression levels were measured by the $2^{-\Delta \Delta C T}$ method. Primer sequences were: HMGB1-F: GCCTTCTTCTTGTTCTGTT, HMGB1-R: TTTCATAGGGCTGCTTG, $\beta$-actin-F: GGAGATTACTGCCCTGGCTCCTAGC, $\beta$-actin-R: GGCCGGACTCATCGTACTCCTGCTT, rnomiR-129-5p-F: CTTTTTGCGGTCTGGGCTTGC, rno-miR129-5p-R: GCAGGGTCCGAGGTATTC, 5S-F: GATCTCGGAAGCTAAGCAGG, 5S-R: TGGTGCAGGGTCCGAGGTAT. $\beta$-actin and $5 S$ were used as internal references for HMGB1 and miR-129-5p, respectively.

\section{Western blotting}

Total protein extraction was performed using RIPA lysis buffer (Beyotime), with 1\% phenylmethanesulfonyl fluoride (PMSF, Beyotime). An enhanced BCA Protein Assay Kit (Beyotime) was used to prepare a standard curve to measure protein concentrations. SDS-PAGE was performed after loading $15 \mu \mathrm{l}$ protein at 15-30 $\mu \mathrm{g}$. Once samples were separated, they were transferred to a PVDF membrane (Invitrogen, Carlsbad, CA, USA). The membrane was blocked in $5 \%$ albumin bovine (BSA, Biosharp), followed by incubation with diluted primary antibodies at $4^{\circ} \mathrm{C}$ overnight: HMGB1 (1:1000, Cell Signaling Technology, Danvers, MA, USA), $\beta$-actin (1:2000, Proteintech, Wuhan, China). After washing steps, secondary antibodies were added and incubated at $37^{\circ} \mathrm{C}$ for 40 min: goat anti-mouse IgG-HRP (1:10000, Proteintech) for $\beta$-actin, and goat anti-rabbit IgG-HRP (1:10000, Proteintech) for HMGB1. Enhanced chemiluminescence (ECL) reagent (7-Sea Biotech, Shanghai, China) was added to membranes to delineate protein signals, after which membranes were scanned and analyzed using Image acquisition and analysis system (WD-9413B, Liuyi, Beijing, China) and Gel-Pro-Analyzer software (Media Cybernetics, USA).

\section{Dual luciferase reporter assay}

$\mathrm{H} 9 \mathrm{c} 2$ cells were used to assess the binding effects of miR129-5p on HMGB1. Cells were seeded in 12-well-plates, and starved $1 \mathrm{~h}$ before transfection. Lipofectamine ${ }^{\mathrm{TM}} 2000$ transfection reagent (Invitrogen) was mixed with HMGB 3'UTR (wt or mutant)-loaded luciferase reporter gene vector pmirGLO (Promega, Wisconsin, USA) and agomiR-129-5p (or agomiR NC) for co-transfection according to manufacturer instructions. The dual luciferase reporter assay kit (Promega) was used to assess relative luciferase activity in each group following manufacturer protocol.

\section{Statistical analysis}

In vivo histological and molecular examinations were performed with six replicates, and all experiments were carried out at least three times. All data were represented as the mean \pm standard deviation (SD). Differences between three or more groups were analyzed using ordinary one-way ANOVA with post-hoc test of Tukey's multiple comparison, and unpaired t-test was adopted to analyze differences between two groups. GraphPad Prism 8 software (San Diego, CA, USA) was used for image/figure processing. A value $p<0.05$ was considered statistically significant.

\section{Results}

\section{miR-129-5p alleviates $I / R$ injury in rat myocardium}

Before establishing the rat I/R model, agomiR-129-5p and agomiR-NC were injected into rat myocardium. The relative expression of miR-129-5p was measured by real-time PCR, confirming that the transfection was effective (Fig. 2A). After $45 \mathrm{~min}$ ischemia and $3 \mathrm{~h}$ reperfusion, LV functional indices showed that I/R-induced significantly increased LVEDP, and reduced LVSP, EF, and FS could be recovered by agomiR-129-5p introduction (Fig. 2B). Serum enzyme activities of myocardial injury biomarker CK and LDH were also evaluated, showing that miR-129-5p upregulation led to a restoration of increased $\mathrm{CK}$ and $\mathrm{LDH}$ activities, after I/R (Fig. 2C). Besides, ELISA of serum levels of inflammatory cytokine TNF- $\alpha$ and IL- $1 \beta$ showed that exogenous miR-129-5p also attenuated the elevated serum TNF- $\alpha$ and IL-1 $\beta$ levels induced by I/R (Fig. 2D). We also quantified infarct size (TTC staining) of rat myocardium groups, and observed that agomiR-129-5p introduction significantly relieved infarct size after I/R injury (Fig. 2E). To further elicit miR-129-5p effects on rat cardiomyocytes after I/R injury, an IF-TUNEL assay was performed to examine cardiomyocyte apoptosis in ischemic penumbra areas. This showed that miR-129-5p reversed much of I/R-induced cell apoptosis (Fig. 2F). Furthermore, the relative expression of HMGB1 and miR-129-5p in each ischemic penumbral area was also assessed (Fig. 2G). Although I/R treatment led to reduced miR-129-5p and enhanced HMGB1 expres- 


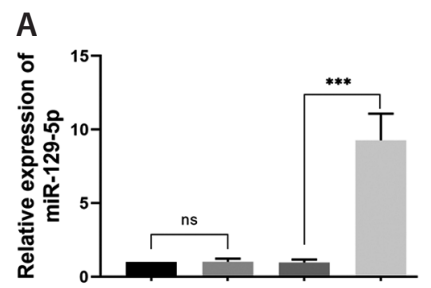

B
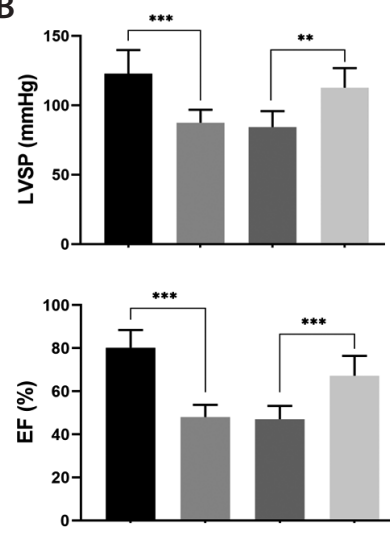

E

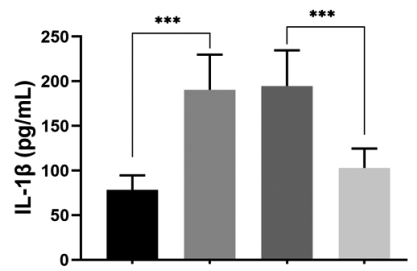

C
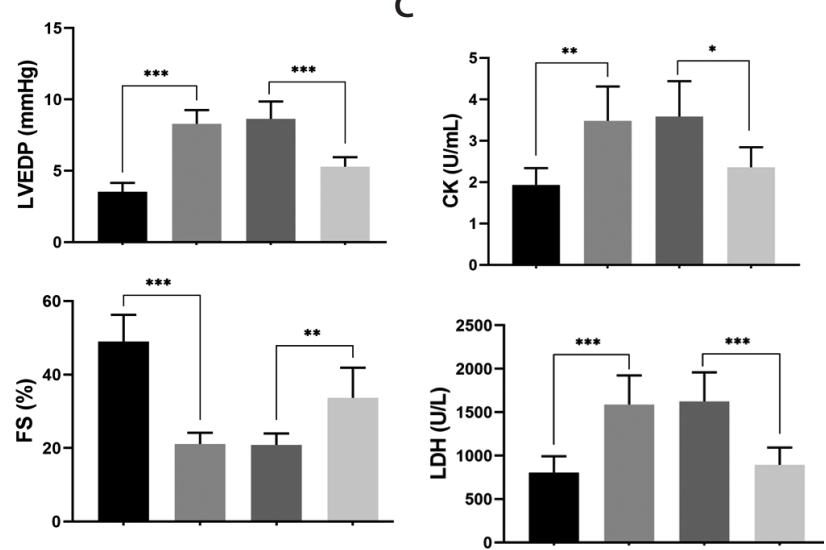

- Sham

I/R

- I/R+agomiR-NC

- I/R+agomiR-129-5p
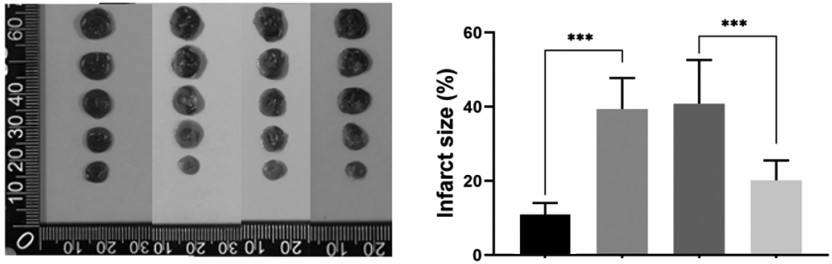

F

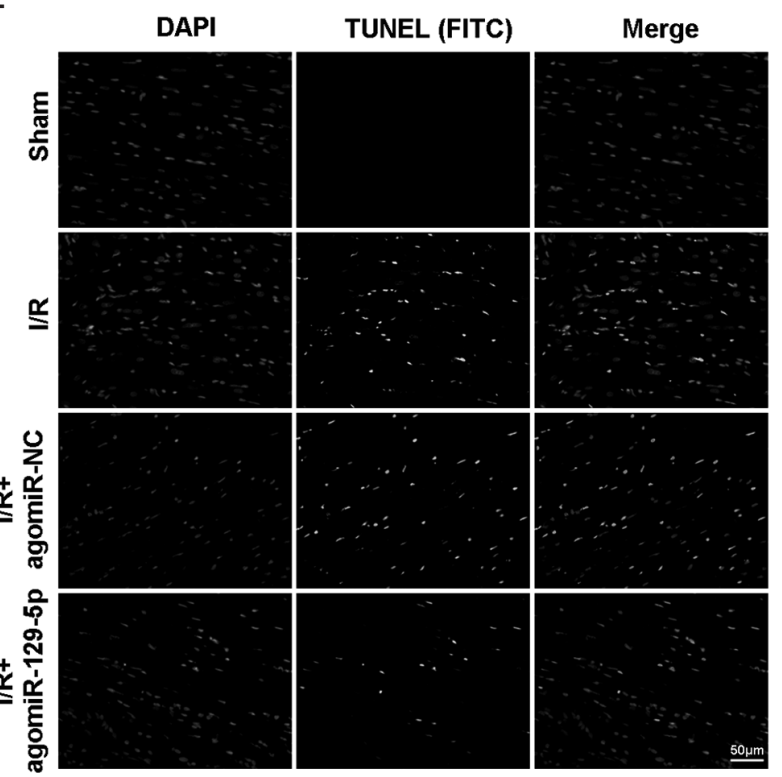

G

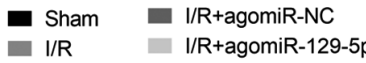
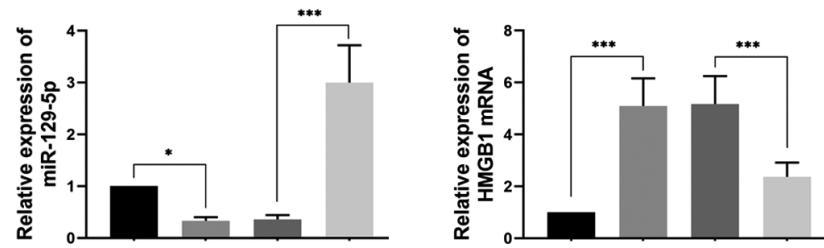

H

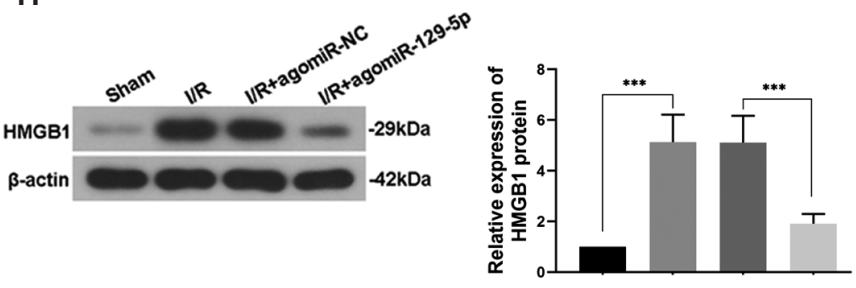

Figure 2. miR-129-5p alleviates I/R injury in rat myocardium. A. Relative expression of miR-129-5p in rat myocardium samples were measured using real-time PCR before I/R modeling. B. Cardiac LV function was determined and represented as LVSP, LVEDP, EF, and FS. C. Enzyme activities of myocardial injury biomarker CK and LDH were checked through their detection kits. D. The inflammatory cytokines TNF- $\alpha$ and IL-1 $\beta$ in myocardium groups were measured by ELISA. E. TTC staining of myocardium tissues in each group. The percentage of infarct size was quantified and measured via IPP. F. The IF-TUNEL assay detected cell apoptosis in myocardium samples. Scale bar represented $50 \mu \mathrm{m}$. G. Real-time PCR quantification of miR-129-5p and HMGB1 relative expression in myocardium groups after I/R injury. H. HMGB1 relative protein levels in myocardium samples through Western blotting assay. ${ }^{\star} p<0.05,{ }^{* *} p<0.01,{ }^{* * *} p<0.001$; $\mathrm{ns}$, no statistical significance. All data were presented as the mean \pm SD. One-way ANOVA was used to analyze data among all four groups. LV, left ventricle; LVSP, left ventricular systolic pressure; LVEDP, left ventricular end-diastolic pressure; EF, ejection fraction; FS, fractional shortening; CK, creatinine kinase; LDH, lactate dehydrogenase; TNF- $\alpha$, tumor necrosis factor- $\alpha$; IL- $1 \beta$, interleukin- $1 \beta$; IPP, Image ProPlus; TUNEL, terminal deoxynucleotidyl transferase dUTP nick end labeling; FITC, fluorescein isothiocyanate; I/R, ischemia-reperfusion. 
sion, agomiR-129-5p administration revealed significantly different effects, increasing miR-129-5p itself and reducing HMGB1 expression. Western blotting also confirmed a similar miR-129-5p effect on HMGB1 expression at the protein level (Fig. $2 \mathrm{H}$ ). Hence, these data suggested that miR-129-5p alleviated myocardium injury and restored infarct perturbations induced by I/R. These observations may also be related to decrease HMGB1 levels.

\section{miR-129-5p affects H9c2 cell viability and apoptosis after $A / R$ injury}

Rat myocardial $\mathrm{H} 9 \mathrm{c} 2$ cells were used as an in vitro cell $\mathrm{A} / \mathrm{R}$ model. Before this, agomiR-129-5p transfection efficiency into $\mathrm{H} 9 \mathrm{c} 2$ cells was evaluated by real-time PCR, showing that the transfection was efficient (Fig. 3A). Cell viability was checked after A/R treatment using CCK-8 assay and showed that miR-129-5p overexpression significantly restored cell viability after A/R injury (Fig. 3B). Annexin V/PI double staining, evaluating cell apoptosis under the same conditions, showed that agomiR-129-5p introduction alleviated cell apoptosis from approximately $23.3 \%$ to $7.8 \%$ after A/R (Fig. 3C). These results collectively indicated that miR-129$5 \mathrm{p}$ restored A/R-induced cell viability, and alleviated cell apoptosis in $\mathrm{H} 9 \mathrm{c} 2$ cells in vitro, which is consistent with the I/R findings.
miR-129-5p targets and negatively regulates HMGB1 in H9c2 cells

To investigate downstream molecule of miR-129-5p in H9c2 cells, the binding of miR-129-5p to HMGB1 was predicted online (TargetScan), and confirmed using dual luciferase reporter analysis (Fig. 4A). The results verified that HMGB1 was a direct target of miR-129-5p in H9c2 cells. Furthermore, relative protein and mRNA expression levels of HMGB1 were measured after H9c2 cells were transfected with agomiR129-5p and antagomiR-129-5p, showing that miR-129-5p overexpression led to reduced HMGB1, whilst miR-129-5p knockdown significantly increased its levels at both translational (Fig. 4B) and transcriptional levels (Fig. 4C). These results suggested that miR-129-5p directly targeted HMGB1 and had a reverse correlation with the HMGB1 expression.

\section{$H M G B 1$ overexpression reverses the effects of $m i R-129-5 p$ in $H 9 c 2$ cells after $A / R$ injury}

To evaluate how HMGB1 affected miR-129-5p regulation in A/R-treated H9c2 cells, cells were co-transfected with agomiR-129-5p and the HMGB1 overexpression plasmid. High co-transfection efficiency was observed, as shown by significantly upregulated miR-129-5p and HMGB1, respectively (Fig. 5A). Cell viability and apoptosis were measured after A/R
A

$$
\begin{aligned}
& \text { Control } \\
& \text { agomiR-NC } \\
& \text { agomiR-129-5p }
\end{aligned}
$$

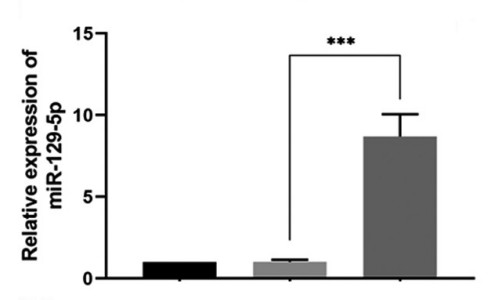

C

$$
\begin{aligned}
& \text { - Control } \\
& \text { A/R } \\
& \text { A/R+agomiR-NC } \\
& \text { A/R+agomiR-129-5p }
\end{aligned}
$$

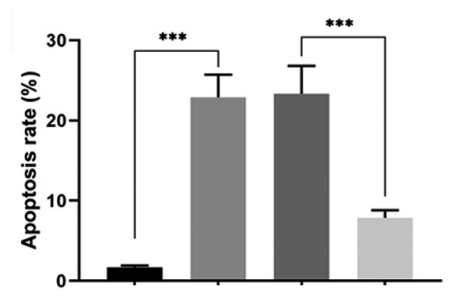

\section{B}

m Contro

- A/R

- A/R+agomiR-NC

- $A / R+$ agomiR-129-5p
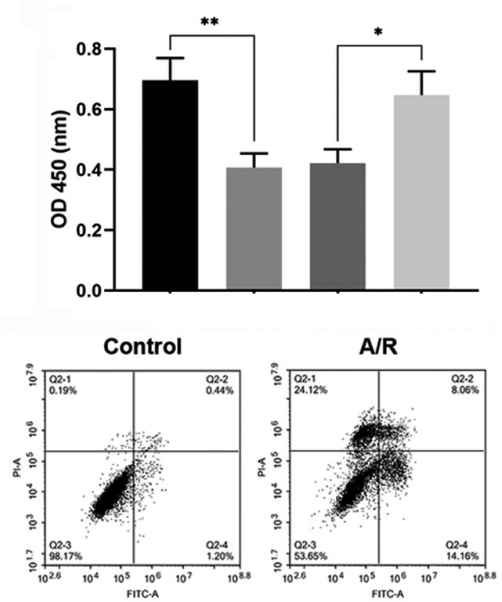

A/R+agomiR-NC

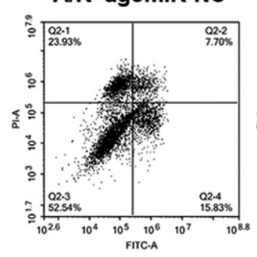

A/R+agomiR-129-5p

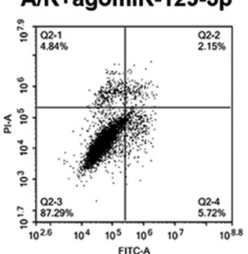

Figure 3. miR-129-5p affects H9c2 cell viability and apoptosis after A/R injury. A. Expression levels of miR-129-5p were assessed after H9c2 cell transfection with agomiR-129-5p. B. Cell viability was measured through CCK- 8 assay after A/R treatment of transfected H9c2 cells. C. Cell apoptosis was detected by Annexin V-FITC/PI double staining and measured using flow cytometry after A/R treatment in each cell group. ${ }^{*} p<0.05,{ }^{* *} p<0.01,{ }^{* * *} p<0.001$. Data were presented as the mean $\pm \mathrm{SD}$. One-way ANOVA was used for data analysis. A/R, anoxiareoxygenation. 
A 3'-UTR HMGB1 (mut): 5'-TACCACTCTGTAATTCGTTTTTA-3'
3'-UTR HMGB1 (wt): 5'-TACCACTCTGTAATTGCAAAAAA-3'
I I I I I I I rno-miR-129-5p: 3'-CGUUCGGGUCUGGCGUUUUUC-5'

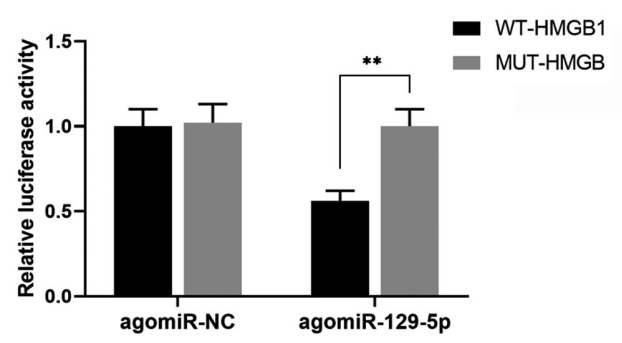

B

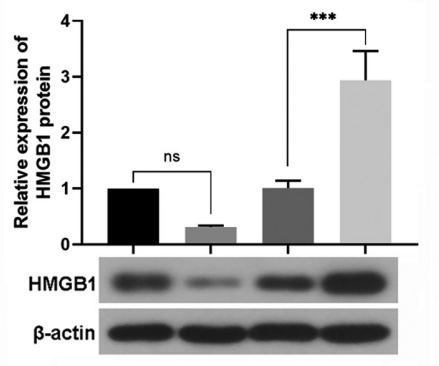

C
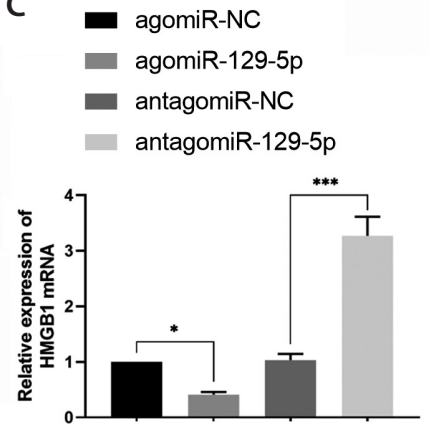

Figure 4. miR-129-5p targets and negatively regulates HMGB1 in H9c2 cells. A. A dual luciferase reporter assay was performed to evaluate miR-129-5p binding to HMGB1 in H9c2 cells. B. H9c2 cells were transfected with agomiR-129-5p and antagomiR-129-5p. Western blotting assay evaluated relative protein expression of HMGB1 in different groups. C. Real-time PCR quantified the relative mRNA expression of HMGB1 in each group. ${ }^{\star} p<0.05,{ }^{* *} p<0.01,{ }^{* *} p<0.001$; ns, no statistical significance. Data were presented as the mean \pm SD. One-way ANOVA was used for data analysis. WT, wildtype; MUT, mutant.

treatment. These data suggested that HMGB1 overexpression decreased the cell viability (Fig. 5B), but enhanced cell apoptosis (Fig. 5C), whilst miR-129-5p was simultaneously overexpressed. Combined with the findings from Figure 3, these data suggested that HMGB1 overexpression reversed the effects of miR-129-5p on rat A/R-injured cardiomyocytes.

\section{Discussion}

Our work investigated the role of miR-129-5p in rat myocardial I/R injury, and whether HMGB1 participated in this miR-129-5p-mediated regulation. To resolve these questions, we established an in vivo rat myocardial I/R model and an
A

$$
\begin{aligned}
& \text { - Control } \\
& \text { agomiR-129-5p+vector } \\
& \text { agomiR-129-5p+HMGB1-OE }
\end{aligned}
$$

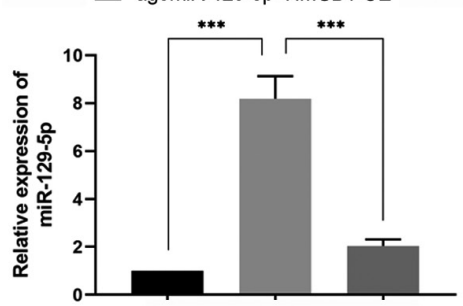

C

A/R+agomiR-129-5p+vector - A/R+agomiR-129-5p+HMGB1-OE

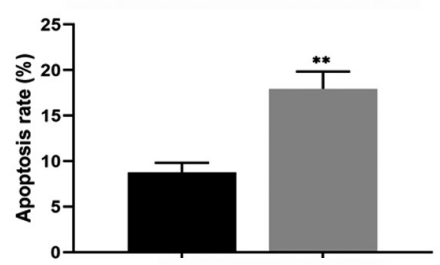

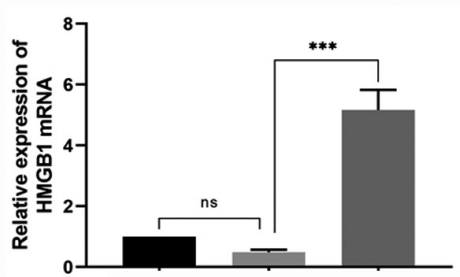

B - A/R+agomiR-129-5p+vector - A/R+agomiR-129-5p+HMGB1-OE
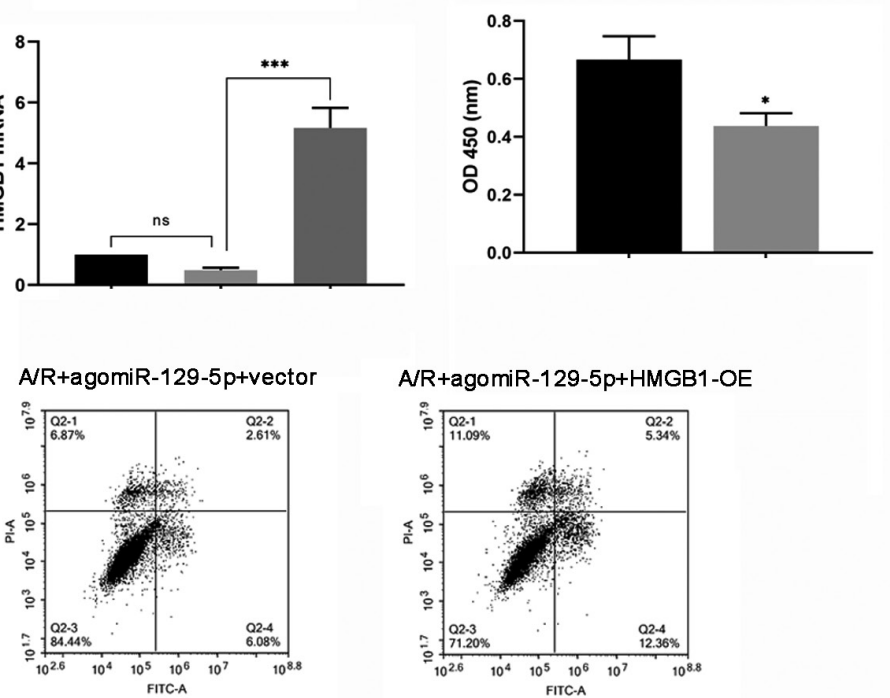

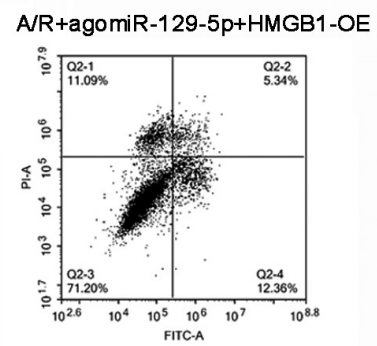

Figure 5. Overexpression of HMGB1 reverses the effects of miR-129-5p in H9c2 cells after A/R injury. A. Rat H9c2 cells were cotransfected with agomiR-129-5p and the HMGB1 overexpression plasmid. Relative expression of miR-129-5p and HMGB1 was assessed before proceeding. B. A CCK- 8 assay evaluated cell viability after co-transfection, and A/R treatment. C. Annexin V-FITC/PI double staining assessed cell apoptosis under the same conditions. ${ }^{\star} p<0.05,{ }^{\star *} p<0.01,{ }^{* * *} p<0.001$; ns, no statistical significance. Data were presented as the mean \pm SD. One-way ANOVA and unpaired t-test methods were used for data analysis. A/R, anoxia-reoxygenation. 
in vitro cardiomyocyte $\mathrm{A} / \mathrm{R}$ model. These models suggested that miR-129-5p alleviated rat myocardial I/R injury by directly binding to $\mathrm{HMGB} 1$, and negatively regulated its expression. Initially, rats with I/R injury of the myocardium showed that miR-129-5p overexpression restored cardiac injury indices and enzyme activities, attenuated inflammatory effects and infarct size caused by $\mathrm{I} / \mathrm{R}$, and also reduced cell apoptosis in rat myocardium tissues. Moreover, overexpressed miR-129-5p recovered cell viability and apoptosis in H9c2 cells after A/R treatment. Although miR-129 has been well-characterized as a tumor suppressor in many cancers (Kouhkan et al. 2016; Liu et al. 2018; Li et al. 2019), its role in hypoxia or ischemia-related diseases has seen an increase in research activity. During ischemic cerebral injury, miR-129 affects neural cell apoptosis through regulating the apoptotic factors, Bcl-2 and caspase-3 (Zhai et al. 2012). Similarly, miR129 also relieves neuro-inflammatory spinal cord damage in a mice model of spinal cord I/R injury (Li et al. 2017). In $\mathrm{H} 9 \mathrm{c} 2$ cells with $\mathrm{H}_{2} \mathrm{O}_{2}$ treatment, miR-129 reduced cell injury and involved in $\mathrm{PI} 3 \mathrm{~K} / \mathrm{AKT} / \mathrm{mTOR}$ signaling pathway, providing a new approach to ischemia heart disease treatment (Zhang et al. 2018). These studies suggested a strong power of miR-129 to modulate several vital signaling circuits related to cellular development in I/R-related diseases. Accordingly, as outlined in this study, its function during I/R injury at the myocardium, suggests that miR-129 may also modify I/R injury at this location, confirming our hypothesis that overexpressed miR-129-5p alleviates myocardial I/R injury to enhance cell viability and suppress cell apoptosis in rats.

The binding effects of miR-129-5p towards HMGB1 were verified in $\mathrm{H} 9 \mathrm{c} 2$ cells, and miR-129-5p was shown to be reversely correlated with the expression of HMGB1. Although agomiR-129-5p introduction in H9c2 cells recovered cell viability and apoptosis after A/R, further HMGB1 overexpression reversed these effects, indicating that HMGB1 has an important role in the miR-129-5p mediated regulation of rat myocardial I/R injury. Actually, the link between miR-129-5p and HMGB1 has already been reported in several cancers and diseases, e.g. colon cancer, gastric cancer, autoimmune encephalomyelitis-related epilepsy, and neuropathic pain, etc. (Liu et al. 2017b; Wu et al. 2018; Wang et al. 2019; Tian et al. 2020). It is noteworthy that miR-129-5p also improves heart function and alleviates oxidative stress and inflammation in rats with chronic heart failure through regulating HMGB1 (Xiao et al. 2019). However, this regulatory mechanism has not been investigated in cardiac I/R injury, until now, where we observed the cardioprotective role of miR-129-5p in heart injury.

As a well-known damage-associated molecular pattern (DAMP) molecule, HMGB1 secreted by necrosis cells, macrophages, or damaged cells mediates strong inflammatory responses through binding to the receptor for advanced glycation end-products (RAGE) or toll-like receptors (such as TLR2, TLR4, and TLR5) (Lee et al. 2015; Das et al. 2016). Previous studies demonstrated that HMGB1-mediated signaling pathways are related to cellular regulation after $\mathrm{I} / \mathrm{R}$ injury in several organs, including the liver, spinal cord and heart (Andrassy et al. 2008; Chen et al. 2011; Zhao et al. 2017; Xie et al. 2018). Andrassy et al. (2008) indicated that HMGB1 levels were increased in the heart after hypoxia or ischemia treatment both in vivo and in vitro, which agreed with our HMGB1 expression data in myocardial I/R injury. Hence, when miR-129-5p directly binds to HMGB1 in cardiomyocytes, it may also inhibit HMGB1-mediated downstream pathways via RAGE or TLRs, to decrease following inflammatory responses, thereby attenuating cellular damages. Although the downstream mechanism of miR-129$5 \mathrm{p} / \mathrm{HMGB} 1$ axis remains to be verified in myocardial I/R injury model, its existence is highly likely, based on literature data and our evidence. Indeed, as miRNAs usually target several genes and there are dozens of predicted targets of miR-129-5p, there may be some other genes acting downstream of miR-129-5p to regulate cardiac I/R injury together, which, however, requires further exploration.

As previously described, myocardial reperfusion therapy has a high risk to cause reperfusion injury in AMI patients, which may induce secondary heart failure or even death. Since non-coding RNAs have been widely implicated in various cellular activities and disease etiologies, non-coding RNA-based gene therapy is considered as a new approach to treat them. In the heart, several miRNAs are dysregulated during cardiac I/R injury, which may be treated as key regulators or biomarkers in these cardiac diseases (Colpaert and Calore 2019; Kura et al. 2020). For example, dysregulation of miR-1, miR-21, and miR-133 was observed in MI heart tissue. Although the precise role of miR-1 is still uncertain in cardiac I/R injury, it is undeniable that miR- 1 is closely related to the myocardial $\mathrm{I} / \mathrm{R}$ processes, and may regulate the adaptive mechanisms after heart injury (Kura et al. 2020). Other important regulators of cardiac I/R injury include miR-21 and miR-133, which are believed to exert cardioprotective functions in MI, mainly through inhibition of apoptosis (Colpaert and Calore 2019; Kura et al. 2020). Combined with our study, down-regulated miR-129 was detected in vivo after I/R injury, when compared with the Sham group. Overexpressed miR-129 ameliorated cardiac injury and also protected cells from apoptosis both in vivo and in vitro, by targeting HMGB1. These observations may underpin a new targeted mechanism for myocardium reperfusion injury-related gene therapy.

In conclusion, we investigated the role of miR-129-5p during rat myocardial I/R injury, both in vivo and in vitro. It was shown that injected agomiR-129-5p alleviated cardiac damage, inflammatory responses, and myocardial infarct size after I/R injury. miR-129-5p directly targeted HMGB1 and negatively regulate its expression in $\mathrm{H} 9 \mathrm{c} 2$ cells. miR-129-5p 
overexpression in A/R treated cardiomyocytes relieved cell apoptosis and restored cell viability, which appeared to be aggravated by further HMGB1 overexpression. Therefore, these results suggest that in rats, miR-129-5p ameliorates cardiac damage induced by $\mathrm{I} / \mathrm{R}$, and protects cardiomyocytes from cell apoptosis by targeting HMGB1. These data provide potential targets for new myocardium reperfusion injury-related therapies.

Acknowledgements. This research was supported by a grant from the Health Commission of Hebei Province (Grant No. 20200309).

\section{Conflict of interests. None.}

\section{References}

Andrassy M, Volz HC, Igwe JC, Funke B, Eichberger SN, Kaya Z, Buss S, Autschbach F, Pleger ST, Lukic IK, et al. (2008): Highmobility group box-1 in ischemia-reperfusion injury of the heart. Circulation 117, 3216-3226 https://doi.org/10.1161/CIRCULATIONAHA.108.769331

Chen KB, Uchida K, Nakajima H, Yayama T, Hirai T, Rodriguez Guerrero A, Kobayashi S, Ma WY, Liu SY, Zhu P, Baba H (2011): High-mobility group box-1 and its receptors contribute to proinflammatory response in the acute phase of spinal cord injury in rats. Spine (Phila Pa 1976) 36, 2122-2129 https://doi.org/10.1097/BRS.0b013e318203941c

Colpaert RMW, Calore M (2019): MicroRNAs in cardiac diseases. Cells 8, 737 https://doi.org/10.3390/cells8070737

Das N, Dewan V, Grace PM, Gunn RJ, Tamura R, Tzarum N, Watkins LR, Wilson IA, Yin H (2016): HMGB1 activates proinflammatory signaling via TLR5 leading to allodynia. Cell Rep. 17, 1128-1140 https://doi.org/10.1016/j.celrep.2016.09.076

Dong LY, Chen F, Xu M, Yao LP, Zhang YJ, Zhuang Y (2018): Quercetin attenuates myocardial ischemia-reperfusion injury via downregulation of the HMGB1-TLR4-NF- $\kappa B$ signaling pathway. Am. J. Transl. Res. 10, 1273-1283 https://doi.org/10.1155/2018/3509091

Fu BC, Lang JL, Zhang DY, Sun L, Chen W, Liu W, Liu KY, Ma CY, Jiang SL, Li RK, Tian H (2017): Suppression of miR-34a expression in the myocardium protects against ischemia-reperfusion injury through SIRT1 protective pathway. Stem Cells Dev. 26, $1270-1282$ https://doi.org/10.1089/scd.2017.0062

Ha M, Kim VN (2014): Regulation of microRNA biogenesis. Nat. Rev. Mol. Cell Biol. 15, 509-524 https://doi.org/10.1038/nrm3838

Hausenloy DJ, Yellon DM (2016): Ischaemic conditioning and reperfusion injury. Nat. Rev. Cardiol. 13, 193-209 https://doi.org/10.1038/nrcardio.2016.5

Kouhkan F, Mobarra N, Soufi-Zomorrod M, Keramati F, Hosseini Rad SM, Fathi-Roudsari M, Tavakoli R, Hajarizadeh A, Ziaei S, Lahmi R, et al. (2016): MicroRNA-129-1 acts as tumour suppres- sor and induces cell cycle arrest of GBM cancer cells through targeting IGF2BP3 and MAPK1. J. Med. Genet. 53, 24-33 https://doi.org/10.1136/jmedgenet-2015-103225

Kura B, Kalocayova B, Devaux Y, Bartekova M (2020): Potential clinical implications of miR-1 and miR-21 in heart disease and cardioprotection. Int. J. Mol. Sci. 21, 700 https://doi.org/10.3390/ijms21030700

Lee DE, Trowbridge RM, Ayoub NT, Agrawal DK (2015): Highmobility Group Box Protein-1, Matrix Metalloproteinases, and Vitamin D in Keloids and Hypertrophic Scars. Plast. Reconstr. Surg. Glob. Open 3, e425 https://doi.org/10.1097/GOX.0000000000000391

Li G, Xie J, Wang J (2019): Tumor suppressor function of miR-129$5 p$ in lung cancer. Oncol. Lett. 17, 5777-5783 https://doi.org/10.3892/ol.2019.10241

Li XQ, Chen FS, Tan WF, Fang B, Zhang ZL, Ma H (2017): Elevated microRNA-129-5p level ameliorates neuroinflammation and blood-spinal cord barrier damage after ischemia-reperfusion by inhibiting HMGB1 and the TLR3-cytokine pathway. J. Neuroinflam. 14, 205 https://doi.org/10.1186/s12974-017-0977-4

Liu AH, Wu YT, Wang YP (2017a): MicroRNA-129-5p inhibits the development of autoimmune encephalomyelitis-related epilepsy by targeting HMGB1 through the TLR4/NF-kB signaling pathway. Brain Res. Bull. 132, 139-149 https://doi.org/10.1016/j.brainresbull.2017.05.004

Liu AH, Wu YT, Wang YP (2017b): MicroRNA-129-5p inhibits the development of autoimmune encephalomyelitis-related epilepsy by targeting HMGB1 through the TLR4/NF-kB signaling pathway. Brain Res. Bull. 132, 139-149 https://doi.org/10.1016/j.brainresbull.2017.05.004

Liu Q, Jiang J, Fu Y, Liu T, Yu Y, Zhang X (2018): MiR-129-5p functions as a tumor suppressor in gastric cancer progression through targeting ADAM9. Biomed. Pharmacother. 105, 420-427 https://doi.org/10.1016/j.biopha.2018.05.105

National Research Council Committee for the Update of the Guide for the C, Use of Laboratory A (2011): The National Academies Collection: Reports funded by National Institutes of Health Guide for the Care and Use of Laboratory AnimalsWashington (DC), National Academies Press (US)

Pu M, Chen J, Tao Z, Miao L, Qi X, Wang Y, Ren J (2019): Regulatory network of miRNA on its target: coordination between transcriptional and post-transcriptional regulation of gene expression. Cell. Mol. Life Sci. 76, 441-451 https://doi.org/10.1007/s00018-018-2940-7

Ranzato E, Martinotti S, Pedrazzi M, Patrone M (2012): High mobility group box protein-1 in wound repair. Cells 1, 699-710 https://doi.org/10.3390/cells1040699

Reed GW, Rossi JE, Cannon CP (2017): Acute myocardial infarction. Lancet 389, 197-210 https://doi.org/10.1016/S0140-6736(16)30677-8

Thygesen K, Alpert JS, Jaffe AS, Chaitman BR, Bax JJ, Morrow DA, White HD (2018): Fourth universal definition of myocardial infarction (2018). J. Am. Coll. Cardiol. 72, 2231-2264 https://doi.org/10.1016/j.jacc.2018.08.1038

Tian J, Song T, Wang W, Wang H, Zhang Z (2020): miR-129-5p alleviates neuropathic pain through regulating HMGB1 expression in CCI rat models. J. Mol. Neurosci. 70, 84-93 
https://doi.org/10.1007/s12031-019-01403-y

Wang S, Chen Y, Yu X, Lu Y, Wang H, Wu F, Teng L (2019): miR129-5p attenuates cell proliferation and epithelial mesenchymal transition via HMGB1 in gastric cancer. Pathol. Res. Pract. 215, 676-682 https://doi.org/10.1016/j.prp.2018.12.024

Wu Q, Meng WY, Jie Y, Zhao H (2018): LncRNA MALAT1 induces colon cancer development by regulating miR-129-5p/HMGB1 axis. J. Cell Physiol. 233, 6750-6757 https://doi.org/10.1002/jcp.26383

Xiao N, Zhang J, Chen C, Wan Y, Wang N, Yang J (2019): miR-129$5 \mathrm{p}$ improves cardiac function in rats with chronic heart failure through targeting HMGB1. Mamm. Genome 30, 276-288 https://doi.org/10.1007/s00335-019-09817-0

Xie T, Li K, Gong X, Jiang R, Huang W, Chen X, Tie H, Zhou Q, Wu S, Wan J, Wang B (2018): Paeoniflorin protects against liver ischemia/reperfusion injury in mice via inhibiting HMGB1TLR4 signaling pathway. Phytother. Res. 32, 2247-2255 https://doi.org/10.1002/ptr.6161

Yellon DM, Hausenloy DJ (2007): Myocardial reperfusion injury. N. Engl. J. Med. 357, 1121-1135 https://doi.org/10.1056/NEJMra071667

Zhai F, Zhang X, Guan Y, Yang X, Li Y, Song G, Guan L (2012): Expression profiles of microRNAs after focal cerebral ischemia/ reperfusion injury in rats. Neural. Regen. Res. 7, 917-923
Zhang H, Zhang X, Zhang J (2018): MiR-129-5p inhibits autophagy and apoptosis of $\mathrm{H} 9 \mathrm{c} 2$ cells induced by hydrogen peroxide via the PI3K/AKT/mTOR signaling pathway by targeting ATG14. Biochem. Biophys. Res. Commun. 506, 272-277 https://doi.org/10.1016/j.bbrc.2018.10.085

Zhao G, Fu C, Wang L, Zhu L, Yan Y, Xiang Y, Zheng F, Gong F, Chen S, Chen G (2017): Down-regulation of nuclear HMGB1 reduces ischemia-induced HMGB1 translocation and release and protects against liver ischemia-reperfusion injury. Sci. Rep. 7, 46272 https://doi.org/10.1038/srep46272

Zhou XM, Liu J, Wang Y, Zhang MH (2018a): Silencing of long noncoding RNA MEG3 enhances cerebral protection of dexmedetomidine against hypoxic-ischemic brain damage in neonatal mice by binding to miR-129-5p. J. Cell. Biochem. 120, 7978-7988 https://doi.org/10.1002/jcb.28075

Zhou XM, Liu J, Wang Y, Zhang SL, Zhao X, Xu X, Pei J, Zhang MH (2018b): microRNA-129-5p involved in the neuroprotective effect of dexmedetomidine on hypoxic-ischemic brain injury by targeting COL3A1 through the Wnt/ $\beta$-catenin signaling pathway in neonatal rats. J. Cell. Biochem. 120, 6908-6919 https://doi.org/10.1002/jcb.26704

Received: March 2, 2020

Final version accepted: June 14, 2020 